\title{
AUTOMATIC EXTRACTION OF RIDGE AND VALLEY AXES USING THE PROFILE RECOGNITION AND POLYGON-BREAKING ALGORITHM
}

\author{
YET-CHUNG CHANG, GWO-SHYH SONG, and SHU-KUN HSU \\ Institute of Oceanography, National Taiwan University, P.O. Box 23-13, Taipei, Taiwan, Republic of China \\ (e-mail:hsu@geophs.oc.ntu.edu.tw)
}

(Received 23 May 1996; accepted 9 July 1997)

\begin{abstract}
For most interpreters working on two-dimensional data or maps, a line-drawing process to depict linear and curvilinear features is often needed. One of the main difficulties in simulating human insight in such a process by computer is that the human eye is able to consider data trends within a wide range on the map. A program based on the profile recognition and polygon-breaking algorithms is introduced in this paper to extract automatically ridge and valley axes. The two algorithms are capable of simulating human performance in the line-drawing process over a range of conditions. The program starts from a profile recognition process which takes all the points close to the possible axes as targets and connects them as a belt of closed polygons. Then, a polygon-breaking process resolves the belt into a continuous line, and a smoothing process further makes the line as smooth as one depicted manually. The program has been applied to the topography of Taiwan and has been successful in extracting ridge and valley systems. It also has shown some potential in solving the problems of line drawing for other purposes and for other kinds of data. (C) 1998 Elsevier Science Ltd. All rights reserved
\end{abstract}

Key Words: Line, Ridge, Profile, Polygon.

\section{INTRODUCTION}

The linear or curvilinear features on many types of maps and digital images, such as the ridge axes on a topographic map, the reflector lines on a seismic profile or the lineaments on a satellite image, are of considerable importance for interpretion. To extract information from maps and images, interpreters generally need a line-drawing process to identify the features. Recently, some automatic line-extraction processes have been developed for several kinds of two-dimensional data. Some examples of these are the segment tracing algorithm (STA) by Koike, Nagano and Michito (1995) for digital images, seismic skeletonization by $\mathrm{Lu}$ and Cheng (1990), and the automated drainage network extraction algorithm by Chorowitz and others (1992).

However, in most situations, such automatic processes have not been widely accepted and the task of line drawing still is done manually. In the authors' opinion, the main reason for this is that the human interpreter can consider data trends within a wide spatial range more effectively than most automatic algorithms suggested to date. It is noted that individual recognition of a "target" line is less important than line continuity according to data trends. The key point in simulating human capabilities is how to design an automatic process that can consider the data trend over a wide spatial range.

With this purpose in mind, some efforts have been made in the development of several automatic processes. For instance, the computation range of the STA has been extended to an $11 \times 11$ matrix (Koike, Nagano and Michito, 1995) and attempts have been made to include three traces into the correlation function of the seismic skeletonization instead of two (Lu and Cheng, 1990). In this paper, the profile recognition and polygon-breaking algorithm (PPA) is proposed, which approaches the requirement of a wide spatial range in a different way.

\section{PROCEDURES OF THE PPA}

The main procedures of the PPA program are shown in Figure 1. A small area within the mountain range of Taiwan Island is taken as an example to test the algorithm (Fig. 2). Both the longitudinal and latitudinal grid intervals of the data set are $0.01^{\circ}$. The figures shown in this paper were plotted with GMT software (Wessel and Smith, 1995; Smith and Wessel, 1990). To reveal the relative importance of the lines, all the segments are subdivided into eight levels which are directly or inversely proportional to their elevations on the 


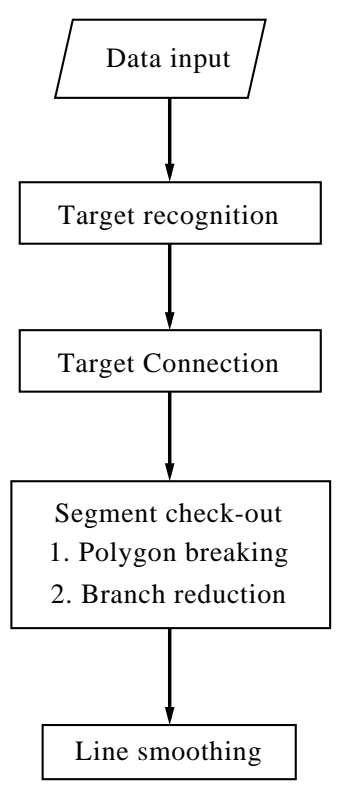

Figure 1. Flowchart of PPA program. figures. For example, the relatively higher ridge axes are plotted as thicker or darker lines.

\section{Target recognition}

The recognition is done with an algorithm that is termed profile recognition. All the points that can be recognized as part of a ridge shape along a profile with limited length are taken as ridge targets. The algorithm first takes the current processed point as the center of the profile. If it can find at least one point lower than the central one on both sides of the profile, the central point is taken as a ridge target. Furthermore, the profile is switched from N-S, NE-SW, E-W to NW-SE to check whether the point is a target or not.

This is a relatively loose recognition process compared to traditional shape recognition because, even if a point is not a local high along any direction, it still is possible to accept it as a ridge target. For example, if a profile length equal to 7 points with elevations of $1,2,3,4,3,2$ and 1 , then not only is



Figure 2. Contours of test area. Background is shaded image based on elevation; higher places are brighter. Map in lower left corner shows position of test area in Taiwan. 


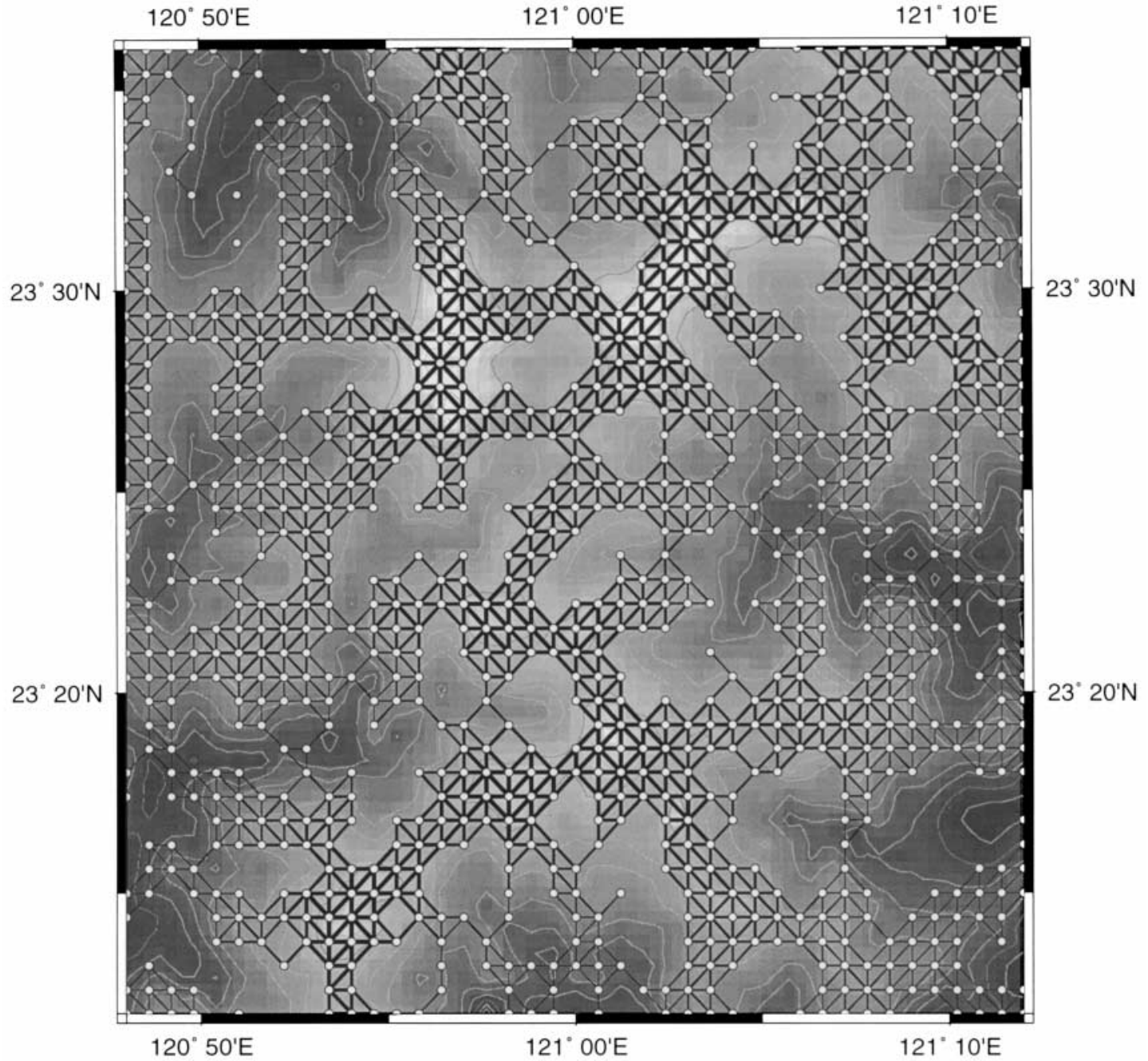

Figure 3. Targets and connections as decided by target recognition and connection processes. Targets are represented by open circles, whereas connections are represented by segments with thickness proportional to elevation.

the central point with elevation of 4 recognized, but so are its two closest neighbors. If, on the other hand, the user takes a profile length equal to 3 , the profile recognition is reduced to a pure shape recognition.
The advantage of profile recognition is that some ambiguous targets, such as a flat bottom of a valley or a flat top of a ridge, also can be confirmed as targets by the points farther beyond the neighbors. This is important to guarantee the continuity of the

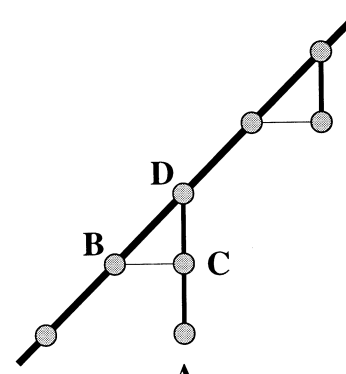

A

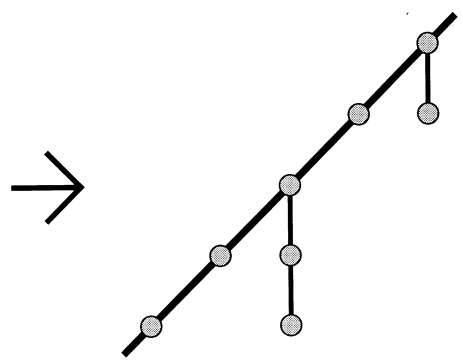

Polygon Breaking



Branch Reduction $=1$

Raw Connection

Figure 4. Simplified sketch for polygon breaking and branch reduction. 


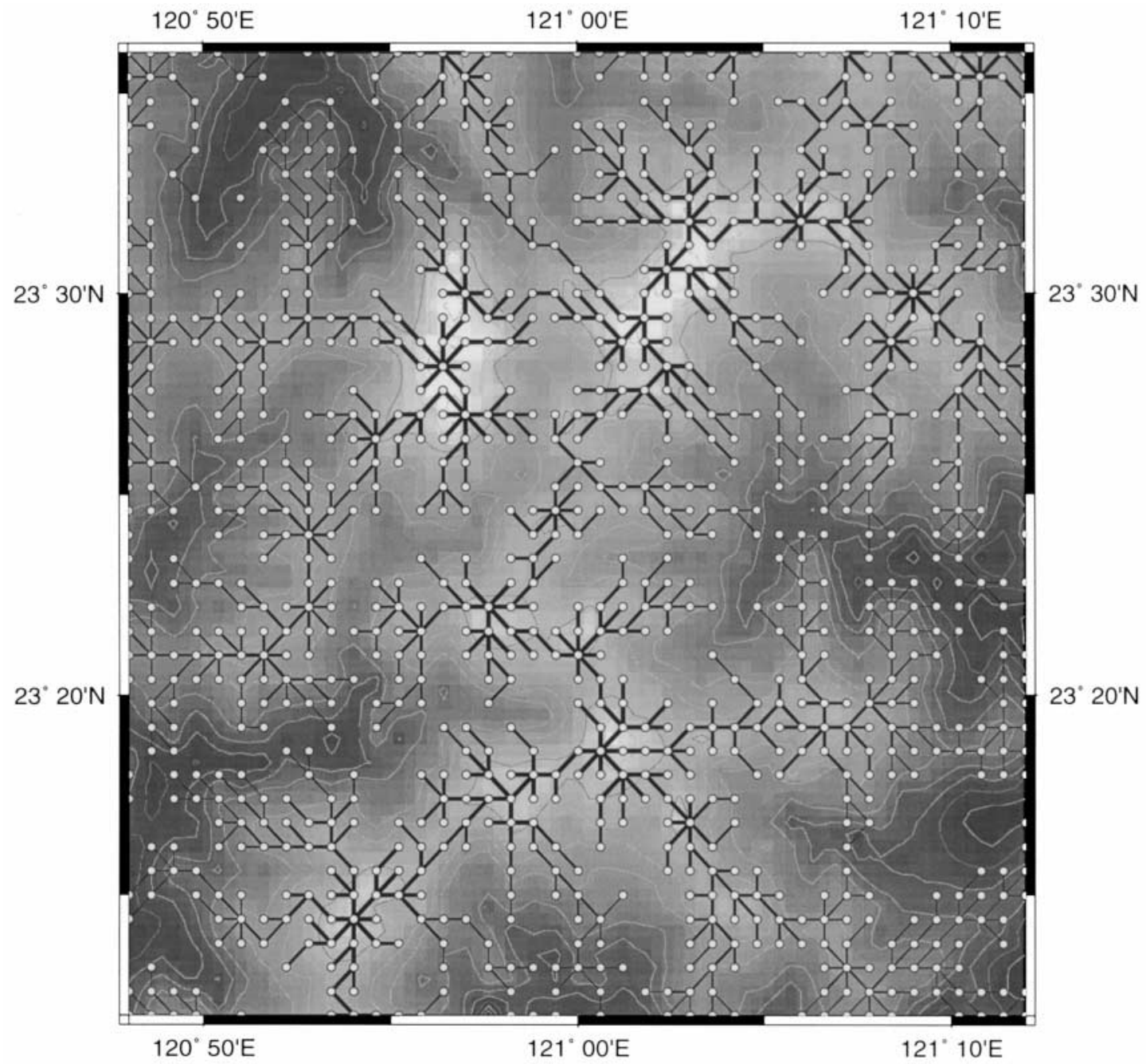

Figure 5. Segments remaining after polygon-breaking process.

axial lines. In this program, if a longer than necessary profile is taken, the result is not greatly changed. Nevertheless, a long profile results in too many targets and tends to waste computation time. Additionally, the branch reduction algorithm which follows, and is designed to reduce the ambiguity caused by profile recognition, might somewhat over-simplify the results. Hence, it is suggested that profile length is selected to be a length just long enough to recognize the possible ambiguous targets on the map.

The open circles in Figure 3 show the results of ridge target recognition following this process in the test area. In this instance, the profile length is taken as 5 points which is long enough to guarantee the continuity of the ridge axes in the test area.

\section{Target connection}

Once target recognition has been accomplished, every two neighboring target points are connected into a segment. For this program, in a 9-point gridded cell, the central point has eight neighbors which are memorized as number 1 for the northern point through number 8 for the other points in a clockwise direction. Under this approach, many diagonal connections cross each other and make many new junction points which are not located on the gridded nodes. When such a situation occurs, the process automatically gets rid of the less important segments; for example, in a ridge search case, the segment with lower elevation is eliminated. The results of target connection in the test area are shown in Figure 3 as segments with different thickness proportional to their elevations.

\section{Segment check-out}

After the process of A and B, it is guaranteed that all possible axes are confirmed within the connected segment groups. In this process, the unfavored segments are cautiously eliminated one by one without breaking the continuity of the lines.

Polygon breaking The polygon breaking is a repeated procedure to check the closed polygon and eliminate the least important segment within each 




Figure 6. Segments remaining after branch reduction.

polygon until no closed polygon of any size remains. The relative importance of segments is determined by their elevation; for instance, for ridge depiction, the segment with lower elevation is less important than segments with higher elevation. After this process, the lines so formed become pure dendritic patterns whose axes are composed of continuous segment groups with many branches instead of a belt of closed polygons.

For each round of polygon breaking in this program, the tracing "tour" starts from one end of the weakest segment which has not been checked previously. When the tracing tour returns to either end of this segment, the tracing stops. Because the possible candidate polygons which may include the segment in question may exist on either side of it, the tracing process is executed in both clockwise and counter-clockwise directions. Referring to the example shown in Figure 4, if the segment B-C is taken as a weak segment and the tracing starts from the target B clockwise, then the tour is B-
$\mathrm{D}-\mathrm{C}$ and a polygon is checked. Once the segment is verified as part of a closed polygon, it is eliminated and the polygon is broken. In the other hand, if the tracing starts from target A, no polygon will be found and the connection between A and $\mathrm{C}$ saved.

A main problem for this process is that both the search for the weakest segment in the data set, and the tracing actions, both take much computation time. To solve part of this problem, the real connection status between targets and the possible tracing routes are handled separately. Once a segment has been checked by the polygon-breaking process, it is taken off of the possible route table, and no tracing tour will pass it again. Additionally, if a segment is verified as an end-segment, it is also removed from the route table. For either of these two situations, the segment is definitely not part of any closed polygon. Such a design can reduce computation time by $50 \%$ without affecting the results of the process. 


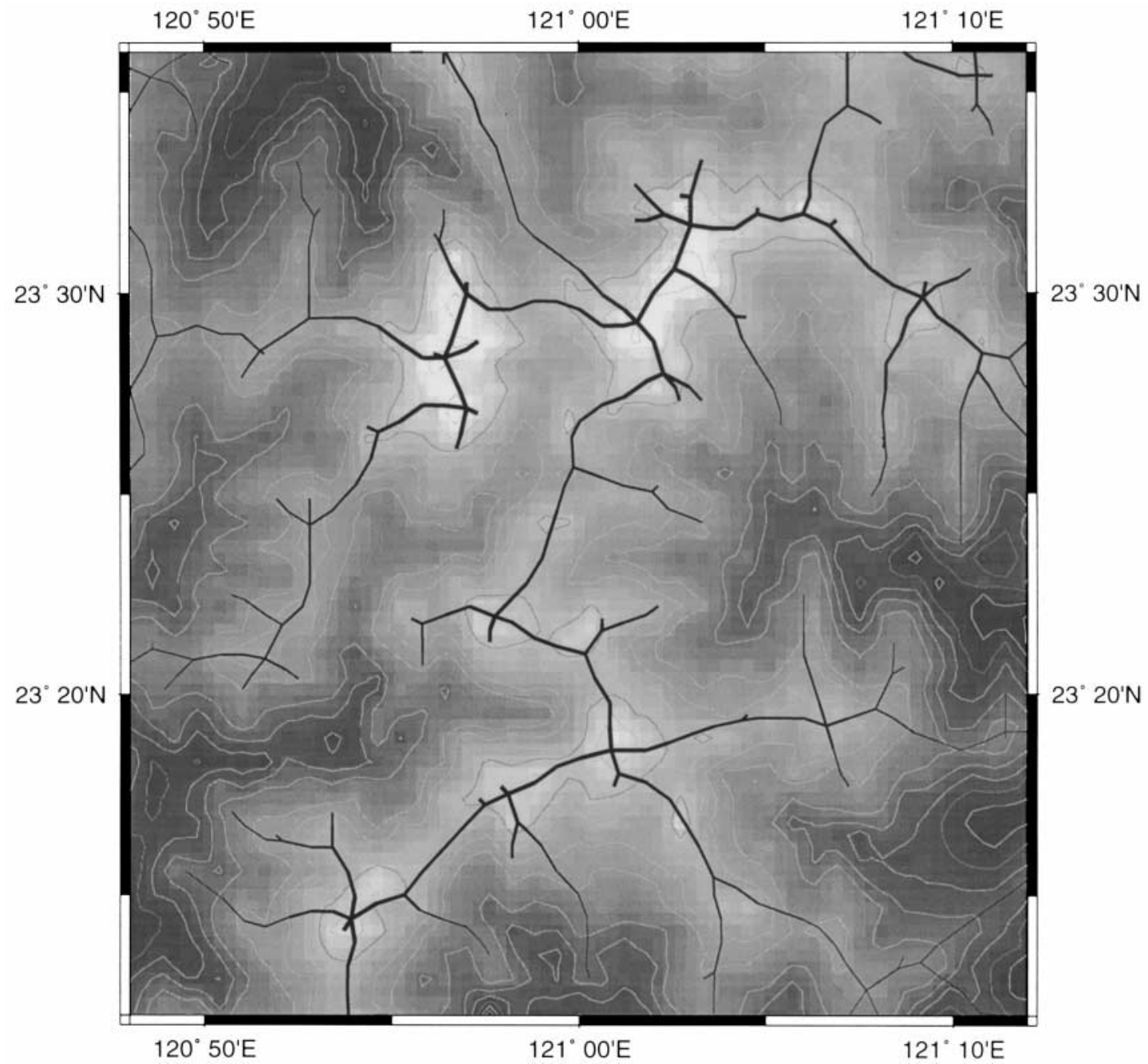

Figure 7. Ridge axes after process of line smoothing.

Branch reduction After polygon breaking, many short branches remain besides the axes (Fig. 5). Most of them are undesirable side effects of profile recognition which generates some over-determined targets around the true axes. As mentioned in the profile recognition section, if a profile length of 7 points is taken, then the width of an axis may be expanded from 1 to 3 . In this instance, after the polygon breaking, it is possible that many pseudobranches shorter than three segments remain beside the true axis. Therefore, in this process all the branches are reduced to one half of the profile length. The result of branch reduction is shown in Figure 6, in which the reduction length is 2 points.

The actions taken for this process are:

1. all the end-points are located;

2. the connections of those end-points are eliminated; and

3. round $=$ round +1 ; if the round is less than the (profile length)/2; step (1) is repeated.

\section{Line smoothing}

This process simply takes the position weighting average of the target and its connected neighbors as its new position. The weight of each target is a value proportional to elevation for ridges, inversely proportional to elevation for valleys. The process can result in targets moving to positions that better match the line trend. Because the new position is an average of the neighboring points and itself, the shifting distance is never more than a grid interval. In this way, the process does not violate the information provided by the gridded data set. Figure 7 shows the results of line smoothing of lines in Figure 6.

\section{The valley axes situation}

If the target lines are the valley axes, the PPA program simply reverses the topography prior to the recognition procedure. Figure 8 shows the results of valley axis extraction of the same area as in previous figures. The profile length also is equal 


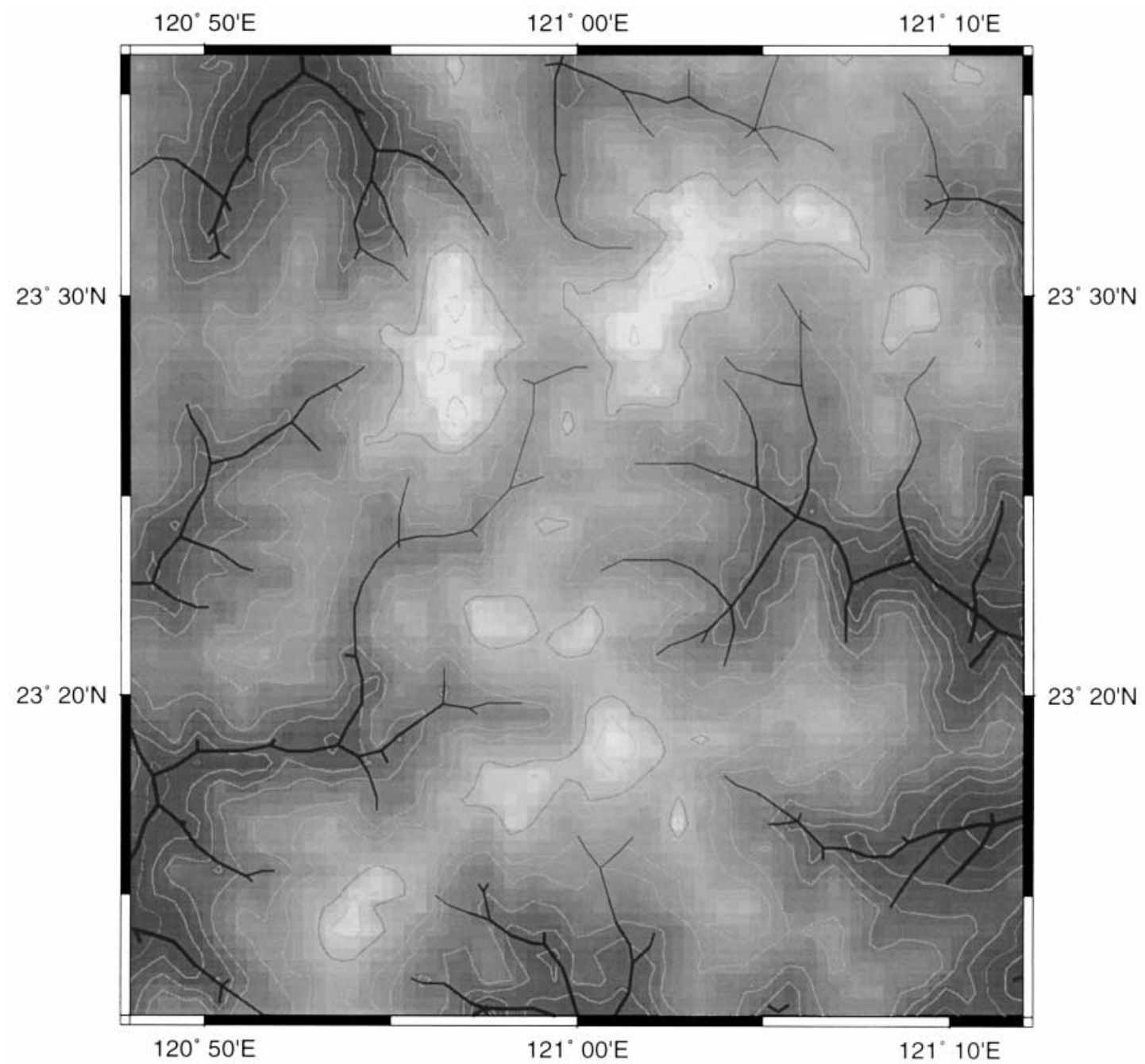

Figure 8. Valley axes extracted by PPA in test area. Profile length for recognition is 5 points.

to 5 points. The ridge and valley axes on the whole of Taiwan Island extracted by the PPA are shown in Figures 9 and 10.

The program is straightforward to use because only the target type and the length of profile recognition need to be defined by the user. Once the data and these two parameters are given, the program can be left to run on its own. An added feature is that the mathematical techniques employed in this program are simple. No matrix computation or any other special function is employed, and even the functions of multiplication or division are seldom used, which makes the program easily understood and modified for other purposes of analysis.

\section{DISCUSSION}

General concept of the automatic line extraction process

The two major goals which need to be accomplished in a line extraction process center upon the target recognition and the decision about connec- tions. The human performance related to this process also can be divided into the consideration of spatial range and context, and the application of domain knowledge according to the data and purpose of analysis. With the aim of making a reasonable line map, these two kinds of human judgment must be applied to both aspects of the work.

Traditionally, the recognition process mostly relies on the domain knowledge; for instance, the ridge should be the local high from a certain direction. On the other hand, the connections are decided by looking for targets at neighboring grid nodes. The problems with this design are:

1. the trend in the data beyond the close neighbors is not considered; and

2. both the rules for recognition and connection are too strict.

This implies that if only one ambiguous target is encountered, a long and continuous line may be broken, thereby ignoring the information that can be seen by the human eye in a wide spatial range. 




Figure 9. Ridge axes on Taiwan with profile length of 5 points for target recognition.

Thus, lines extracted by this method tend to be discrete and short and, therefore, are not favored by most interpreters. To solve this problem, some additional procedures generally are needed which reconsider line continuity based on the discrete lines and merge or reconnect some of the short lines (e.g. Raghavan and others, 1995).
In this program, a different approach is selected to obtain the more desirable continuous line patterns. First, a loose recognition algorithm which also includes some ambiguous targets is used. Then, the over-determined targets and connections are cleared by the polygon breaking and branch reduction process step-by-step without breaking the 


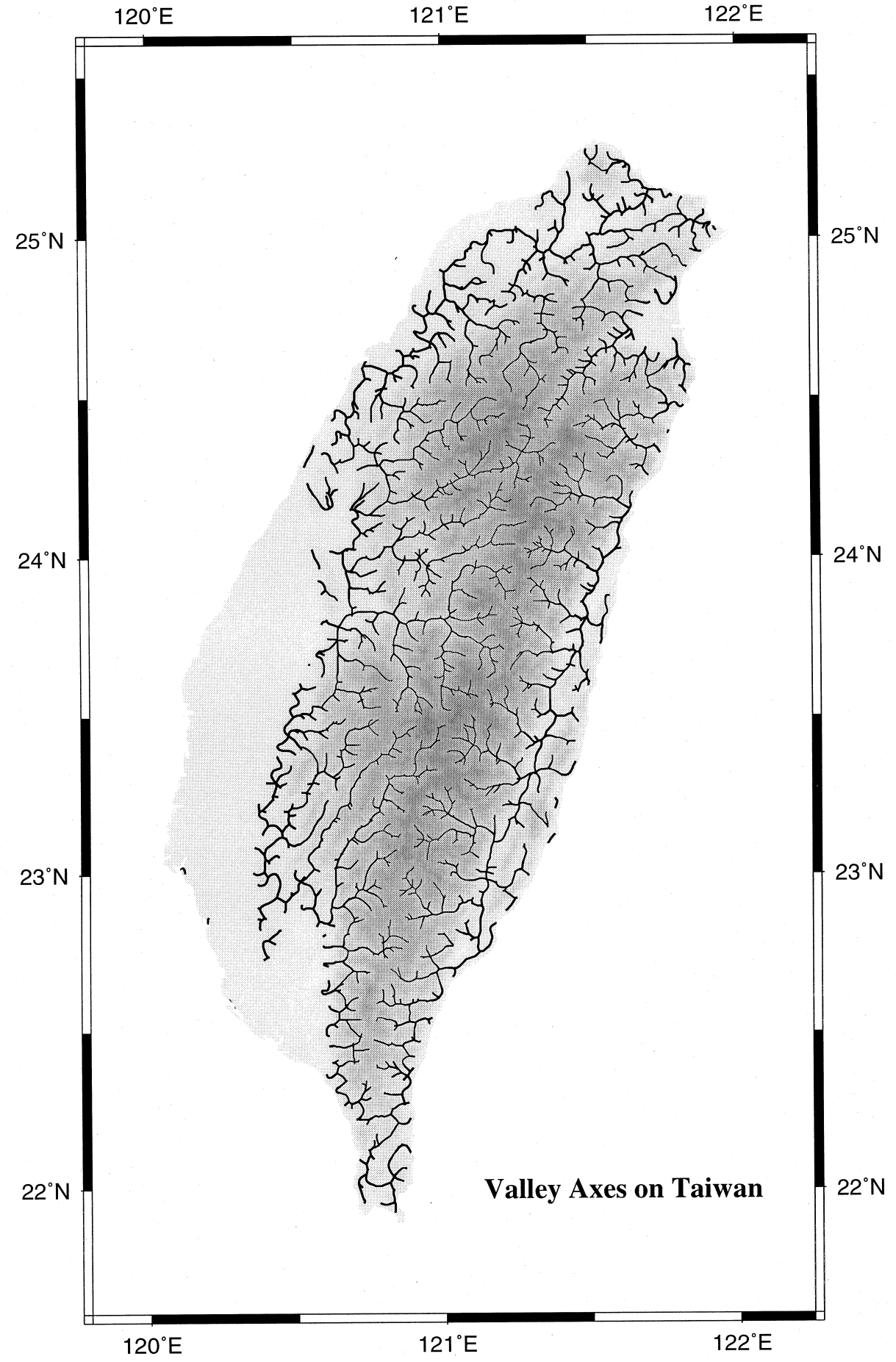

Figure 10. Valley axes on Taiwan with profile length of 5 points for target recognition.

line continuity. This way is more reasonable than traditional merging or amending designs, because broken lines may be linked by ambiguous targets which still are recognized by the looser and more flexible criterion. On the other hand, a merging process considers only the continuity of a line, which may cause a definitely non-target point to be included in a target line.

\section{About the wide spatial range effects}

To illustrate the benefits of the algorithms, the profile recognition and polygon breaking is reduced to a pure shape recognition (profile length $=3$ ) and applied on the test area of the previous figures. Results after the segment check-out processes are shown in Figure 11. It is supposed that most read- 


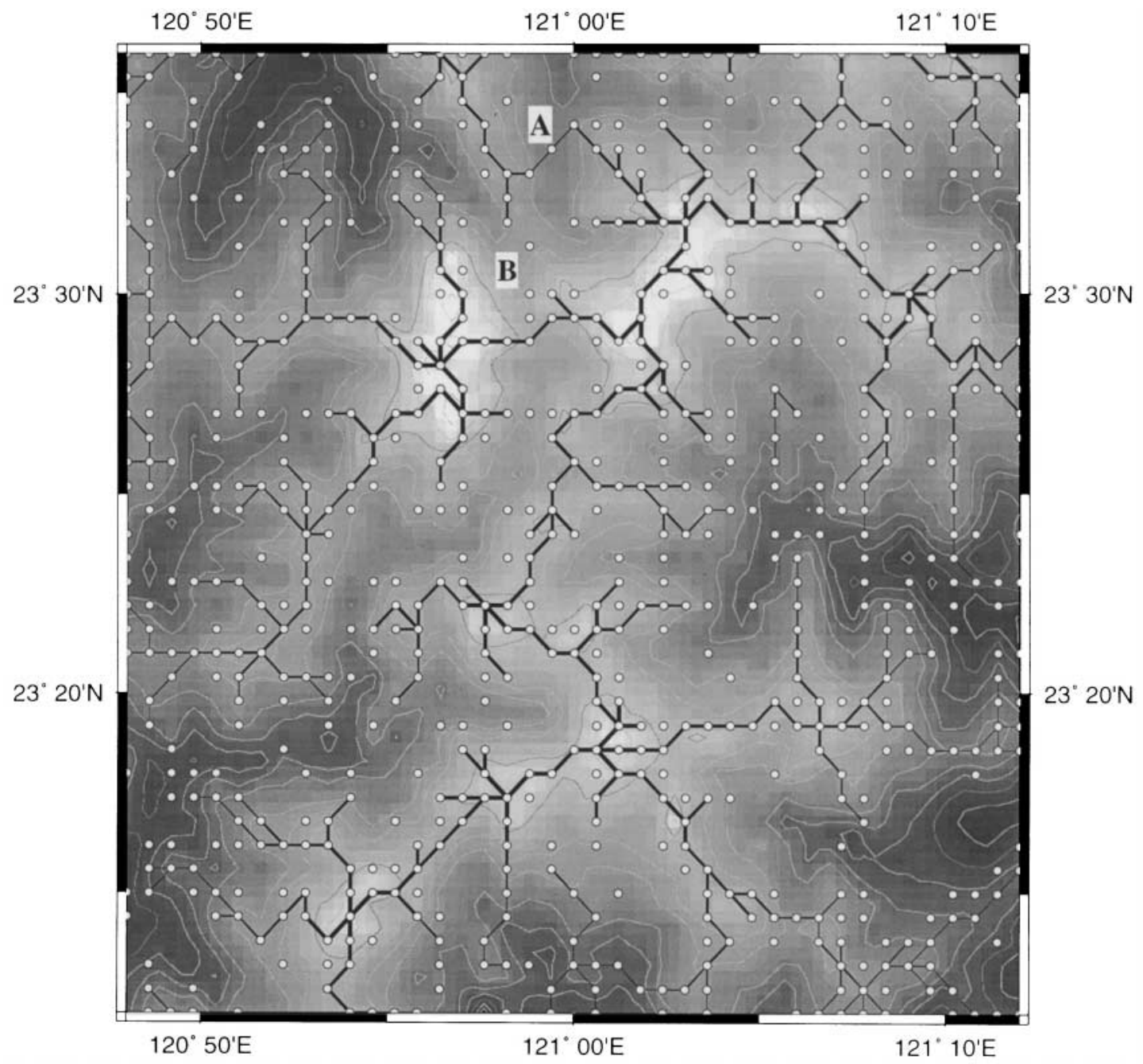

Figure 11. Segments remaining after polygon breaking and branch reduction processes. Profile length for recognition is 3 points.

ers will expect the line to be connected at site B on Figure 11 rather than site A. However, the computer did not make any mistake! The disconnection at site B is simply because the topography is "flat" with respect to the neighboring data points at that location. On the other hand, the connection at site A is because the point is recognized as a target with respect to the neighboring data. In fact, the reason for favoring the connection at $\mathrm{B}$ rather than $\mathrm{A}$ is that a zone wide enough to include both $\mathrm{A}$ and $\mathrm{B}$ simultaneously can be considered by a human interpreter. If the eye and brain could not consider a wide zone, then the human decision would be the same as the one made by an automatic process.

In this program, profile recognition is a procedure which recognizes the target based on a wide zone extending beyond the immediate neighbors. If a target cannot be confirmed by the immediate neighbors, then the data points farther away provide more information for recognition. For example, if the profile length is extended from three to five, both sites $\mathrm{A}$ and $\mathrm{B}$ are connected (see Fig. 3). On the other hand, the polygon-breaking step helps to disconnect polygons from smaller ones to a larger one. When the remaining polygon is wide enough to include both the connections at sites $\mathrm{A}$ and $\mathrm{B}$, their relative importance is taken into consideration simultaneously and the connection at $\mathrm{A}$ is cancelled (see Fig. 5). In other words, the program can consider the property of many segments distributed over a wide spatial range on the map and make a single decision of connection or not by the polygon-breaking process.

\section{About the valley axes extraction}

It is noted that the valley axes pattern decided by the PPA is similar to a drainage system (see Fig. 8). The main difference of the results between the PPA and the traditional automated drainage extraction programs (e.g. Chorowitz and others, 1992; O'Callaghan and Mark, 1984) is that the latter generally fill the depressions to guarantee continuous 
downslope gradients, but the PPA simply depicts the topographic low axes. However, if some criteria to characterize the drainage were included, the program also might provide an alternative to existing methods of extracting the drainage system.

\section{CONCLUSION}

A new concept that involes consideration of a wide spatial range is proposed to extract topographic information automatically. To simulate the process of human interpretation, the proposed algorithm employs a relatively loose profile recognition criteria to include both clear and ambiguous targets and connect them as belts of closed polygons. An iterative and rigorous step of polygon breaking is used to eliminate the least important segments without breaking line continuity. The remaining segments are finally smoothed by shifting the connected targets lines to positions guided by trends in the data.

\section{REFERENCES}

Chorowitz, J., Ichoko, C., Riazanoff, S. and Kim, Y. J. (1992) A combined algorithm for automated drainage network extraction. Water Resources Research 28, 1293-1302.

Koike, K., Nagano, S. and Michito, O. (1995) Lineament analysis of satellite images using a segment tracing algorithm (STA). Computers \& Geosciences 21(9), 10911104.

Lu, S. Y. and Cheng, Y. C. (1990) An iterative approach to seismic skeletonization. Geophysics 55(10), 13121320.

O'Callaghan, J. F. and Mark, D. M. (1984) The extraction of drainage networks from digital elevation data. Computer Vision, Graphics and Image Processing 28, 323-344.

Raghavan, V., Matsumoto, S., Koike, K. and Nagano, S. (1995) Automatic lineament extraction from digital images using a segment tracing and rotation transformation approach. Computers \& Geosciences 21(4), 555591.

Smith, W. H. F. and Wessel, P. (1990) Gridding with continuous curvature splines in tension. Geophysics 55, 293-305.

Wessel, P. and Smith, W. H. F. (1995) New version of the Generic Mapping Tools released. EOS Transactions American Geophysical Union 76, 329. 